\title{
SPEED-GRADIENT CONTROL OF PASSING THROUGH RESONANCE IN ONE- AND TWO-DIMENSIONAL MOTION
}

\author{
Olga Tomchina $^{1}$, Dmitry Tomchin ${ }^{2}$, Alexander Fradkov ${ }^{2}$ \\ 1) St.Petersburg Machine Building Institute, \\ 14, Poliustrovskii ave, St.Petersburg, Russia \\ ${ }^{2)}$ Institute for Problems of Mechanical Engineering \\ 61, Bolshoy ave. V.O., Saint Petersburg 199178, Russia \\ dimatom@mail.wplus.net
}

\begin{abstract}
An approach to control of passing through resonance zone based on speedgradient energy control of two subsystems (rotor and support) is presented. Two typical problems of passing through resonance for one- and two-dimensional motion of support are posed and analyzed by computer simulation. The control algorithms based on speedgradient method and averaging allow to significantly reduce the required level of the controlling torque. The algorithms have small number of design parameters. Compared with the known algorithms the proposed ones are more simple for design and exhibit stronger robustness properties. Copyright $@ 2005$ IFAC
\end{abstract}

Keywords: oscillations, nonlinear control, speed-gradient method.

\section{INTRODUCTION}

Vibrational units with unbalanced (eccentric) rotors are widely used in the industry. An important problem for their design is to reduce the maximum power of the driving motor achieved during the spin-up mode (Blekhman, 2000). The decrease of the spin-up power leads to decrease of nominal power and, therefore to decrease of the weight and the size of the motor. Another problem is in that in order to obtain the desired mode of vibration it is necessary to control the rotor speed in a broad range including both pre-resonance and postresonance regions. The problem of passage through resonance becomes more difficult when the power of the motor decreases. Therefore solution of both problems is important for developing vibrational equipment with improved technological characteristics.

The key idea to reduce the spin-up power of the unbalanced rotor is to swing the rotor during the spin-up period by feedback control. Different approaches to solve the problem were proposed (Malinin and Pervozvansky, 1993; Kel'zon and Malinin, 1992; Rand, et. al., 1992; Kinsey, et. al., 1992). In (Malinin and Pervozvansky, 1993; Kel'zon and Malinin, 1992) the control law for oscillating eccentric rotor was proposed based on optimal design using Pontryagin maximum principle. However the practical implementation of this law is difficult because of complex calculations required for solving non-linear optimal control problem. In (Rand, et. al., 1992; Kinsey, et. al., 1992) the phenomenon of resonant capture arising in the dynamics of dual-spin spacecraft was considered. However the control design proposed in those papers is based on the averaged equations depending on coordinates which represent composite functions of initial variables. The design procedure requires calculation of the averaged angle coordinate of transformed model which is a very labor-consuming process.

A new approach to feedback control of passage through resonance was proposed in (Tomchina, 1997; Tomchina and Nechaev, 1999) for onedimensional motion of support based on the speedgradient method with energy-based goal functions (Fradkov, 1996). As it was shown in (Fradkov, 1996) the speed-gradient algorithms for energy control of conservative systems allow to achieve an arbitrary energy level by means of arbitrarily small level of control power. Using this approach for systems with losses allows to spend energy only to compensate the losses, and to reduce the power of 
driving motor significantly. However, reduction of the motor power for systems with several degrees of freedom may increase the influence of resonance and lead to appearance and amplification of Sommerfeld phenomenon and capture (Blekhman, 2000). The case of plane motion was studied in (Malinin and Pervozvansky, 1993), where the controller was designed using optimal control technique, see also (Kel'zon and Malinin, 1992).

In this paper an approach to the problem of controlled passage through resonance zone for multidimensional systems is presented. The control algorithms based on speed-gradient method and averaging are proposed. Applications to onedimensional system (TORA example) and to a twodimensional system (plane motion of one-rotor vibrational unit) demonstrate efficiency of the approach. The proposed algorithms allow to significantly reduce the required level of the controlling torque. The efficiency of the algorithms is studied by means of computer simulation.

\section{PROBLEM STATEMENT}

We use the standard Euler - Lagrange form of mechanical systems description

$$
M(q) \ddot{q}+C(q, \dot{q})+G(q)=u
$$

where $u$ is $n \times 1$ vector of control torques; $q, \dot{q}, \ddot{q}$ are $n \times 1$ vectors of generalized coordinates, velocities and accelerations correspondingly; $M(q)$ is $n \times n$ inertia matrix; $C(q, \dot{q})$ is $n \times 1$ vector of Coriolis and centrifugal forces; $G(q)$ is $n \times 1$ vector of gravity forces; $n$ is the number of the plant degrees of freedom. The losses (Rayleigh dissipation function) are not taken into account.

We will also use the Hamiltonian form which is convenient for the purpose of controller design. The controlled plant equations in Hamiltonian form are as follows:

$$
\dot{p}=-\left(\frac{\partial H}{\partial q}\right)^{T}+B u, \quad \dot{q}=\left(\frac{\partial H}{\partial p}\right)^{T},
$$

where $p, q \in \mathfrak{R}^{n}$ - are generalized coordinates and momenta; $H=H(p, q)$ - Hamiltonian function (total energy of the system); $u=u(t)-m$ dimensional input vector (generalized forces), $B$ is $m \times n$ matrix, $m \leq n$.

Formalize the main control objective as approaching the given energy level of the unforced system

$$
H(p(t), q(t)) \rightarrow H^{*}, \text { when } t \rightarrow \infty .
$$

Introduce the objective function as follows

$$
Q(p, q)=1 / 2\left[H(p, q)-H^{*}\right]^{2} \text {. }
$$

Then the objective (3) can be reformulated as

$$
Q(q(t), p(t)) \rightarrow 0, \text { when } t \rightarrow \infty \text {. }
$$

Let the additional inequality constraint be given:

$$
H_{1}(p(t), q(t)) \leq \Delta,
$$

where $H_{1}$ is kinetic energy of first subsystem:

$$
H_{1}(p(t), q(t))=\frac{1}{2} \dot{q}_{1}^{T} M_{11}\left(q_{1}\right) \dot{q}_{1},
$$

where $q_{1}$ is $n_{1}$-vector of generalized coordinates of the first subsystem; $M_{11}\left(q_{1}\right)$ is the corresponding $n_{1} \times n_{1}$ - inertia submatrix. The problem is to design the control algorithm for the system (1) or (2), ensuring the objective (3) under constraint (6).

\section{DESIGN OF CONTROL ALGORITHMS}

The idea of solution is based on separation of motions near the resonance (Rand, et. al., 1992; Pechenev, 1986). Using the averaging method allows to detect two components with different rate in the system motions near resonance. In (Pechenev, 1986) the more slow oscillatory component was called the slow motion (in contrast to the "superslow" change of the averaged velocity before and after the resonance. It was shown in (Pechenev, 1986) by means of vibrational mechanics approach based on the averaging method that the oscillations of the slow component may be described by the 1-DOF pendulum-like equation. For passing through the resonance it was suggested in (Tomchina, 1997) to swing the slow motions up until the total energy $H$ achieves the prescribed level and the energy of the subsystem $H_{1}$ becomes sufficiently small. Also it was suggested to apply the speed-gradient (SG) algorithm for swinging the system

$$
u=-\gamma \Psi\left(\nabla_{u} \dot{Q}\right)
$$

where $\dot{Q}$ is derivative of $Q$ with respect to the equations (2), $\nabla_{u}$ stands for the gradient in $u$, $\gamma>0$ is gain coefficient and value of the vectorfunction $\Psi(z)$ forms an acute angle with the vector $z$, i.e. $\Psi(z)^{T} z>0$ for $z \neq 0$. For pendulum-like 1-DOF systems SG algorithms look as follows:

$$
\begin{gathered}
u=-\gamma\left(H-H^{*}\right) \dot{\psi}, \\
u=-\gamma \operatorname{sign}\left[\left(H-H^{*}\right) \dot{\psi}\right], \\
u=-\gamma \operatorname{sgn}\left[\left(H-H^{*}\right) \dot{\psi}\right],
\end{gathered}
$$

where $\psi$ is the oscillating generalized coordinate, $\operatorname{sign} x=1$, if $x \geq 0, \quad \operatorname{sign} x=-1$, if $x<0$; $\operatorname{sgn} x=1$, if $x \geq 0, \operatorname{sgn} x=-1$ if $x<0$. For our purposes the above algorithms or their modifications can be used where $\psi$ is chosen as the slow variable near the resonance. To implement the algorithms (9) - (11) it is necessary to measure the velocity $\dot{\psi}$ of the slow variable $\psi$.

In this paper it is suggested to obtain $\psi$ as the averaged value of the fast variable velocity $\omega=\dot{\theta}$, i.e. $\dot{\psi}$ as the averaged value of $\ddot{\theta}$. The averaging 
is performed by the first order low-pass filter ("dirty differentiator")

$$
T \dot{\psi}(t)=-\psi(t)+\dot{\theta}(t)
$$

Time constant $T$ should allow to suppress fast motion, i.e. its value should lie in the interval between the periods of fast and slow oscillation.

It is also proposed to switch on the speed-gradient algorithms (9) - (11) with filter (12) when the first subsystem energy is large (inequality (6) violates) and to switch to the conventional control $u(t) \equiv \gamma>0 \quad$ when (6) holds. Additionally, to achieve better performance, the current value of the energy $H_{1}$ is subject to filtering.

\section{EXAMPLE 1. TRANSLATIONAL OSCILLATOR - ROTATIONAL ACTUATOR}

Consider the problem of controlling translational oscillator - rotational actuator (TORA), consisting of a support (cart) attached to a wall by a spring (Fig.1). A rotating eccentric mass (debalanced rotor) connected to the cart is actuated by a DC motor. Initially this system has been used as a simplified model to study the resonance capture (Sommerfeld) phenomenon (Rand, et. al., 1992; Ewan-Ivanovski, 1976). Recall that the capture phenomenon represents the failure of a rotating mechanical system to be spun up by a torquelimited rotor to a desired rotational velocity due to its resonant interaction with another part of the system (Ewan-Ivanovski, 1976), see also Fig.2. Similar system (RTAC - Rotational/Translational ACtuator) was proposed by D.Bernstein with coworkers (Wan, et. al., 1994; Bupp, et al., 1998) as a benchmark example for nonlinear control and later was called TORA, see (Jankovic, et. al., 1996). However, in the existing control related results only stabilization problem was addressed.

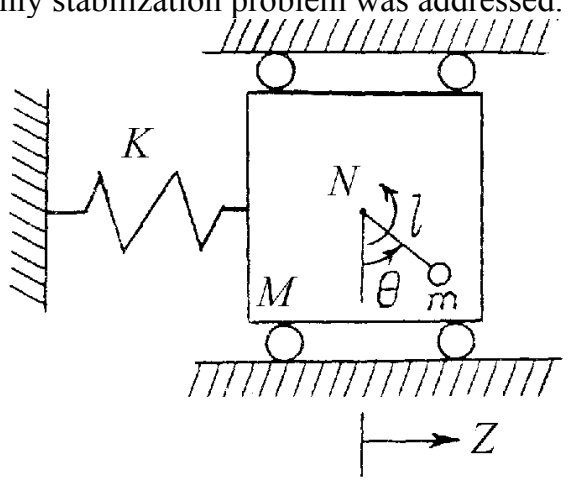

Fig. 1. TORA schematics (horizontal plane).

We pose the problem as spinning the system up to the desired energy level under restriction on the energy of its specified subsystem. The goal is to achieve the desired average angular velocity of the motor under constraint imposed on the translational oscillations of the cart. The motor torque is assumed to be a control variable. The model of the system is as follows

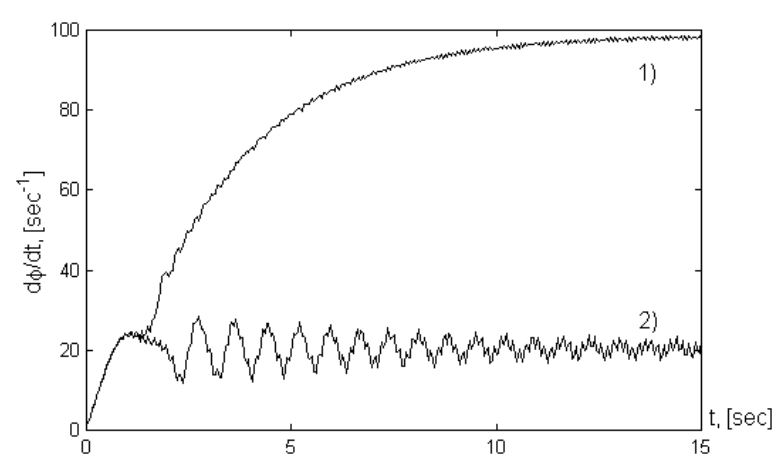

Fig. 2 Conventional control $u(t) \equiv \gamma_{0}$. 1) $\gamma_{0}=0.49$ - capture, 2) $\gamma_{0}=0.50$ - passage.

$$
\begin{gathered}
(M+m) \ddot{z}+k_{1} \dot{z}+m l\left(\ddot{\theta} \cos \theta-\dot{\theta}^{2} \sin \theta\right)+k z=0, \\
\ddot{J} \theta+k_{\theta} \dot{\theta}+m l \ddot{z} \cos \theta=u,
\end{gathered}
$$

where $z$ is the displacement of the cart from its equilibrium position, $\theta$ is rotational angle of the rotor, $u=N(t)$ is motor torque (control variable).

The system has the state vector $x=(z, \dot{z}, \theta, \dot{\theta})^{T} \in \mathfrak{R}^{4}$. The total energy of the system is as follows

$H=\frac{1}{2}(M+m) \dot{z}^{2}+m l \dot{z} \dot{\theta} \cos \theta+\frac{1}{2} m l^{2} \dot{\theta}^{2}+\frac{1}{2} k z^{2}$.

The model (13) and the energy (15) can be expressed also in the Hamiltonian form. We want to achieve the control goal

$$
Q(x(t)) \rightarrow 0 \text {, }
$$

where $Q=\left(H-H^{*}\right)^{2} / 2$ and $H^{*}$ is the desired energy level under the constraint $H_{1}(x(t)) \leq \Delta$, where $\Delta>0$ and $H_{1}$ is the kinetic energy of the cart $H_{1}(x)=M \dot{z}^{2} / 2$. For control the modification of SG algorithm (11), (12) was chosen:

$$
u= \begin{cases}\gamma, & \text { if } \quad\left(H-H^{*}\right) \dot{\psi}<0, \\ 0, & \text { else, }\end{cases}
$$

where $\psi$ is the slow variable. Note that the filter (12) allows to obtain the sign of $\dot{\psi}(t)$ just comparing the values of $\dot{\theta}(t)$ and $\psi(t)$.

The numerical investigation of the system performance for different values of controller parameters $\gamma$ and $T$ was carried out by means of MATLAB software. The system parameters were taken as in (Blekhman, et. al., 1999): $J=0.014$ $\left[\mathrm{kg} \cdot \mathrm{m}^{2}\right], M=10.5[\mathrm{~kg}], m=1.5[\mathrm{~kg}], l=0.04[\mathrm{~m}]$, $k_{\theta}=0.005 \quad[\mathrm{~J} \cdot \mathrm{sec}], \quad k=5300 \quad[\mathrm{~N} / \mathrm{m}], \quad k_{1}=5$ $[\mathrm{kg} / \mathrm{sec}]$. For comparison the time history of angular velocity of the rotor driven by constant torque $u(t)=\gamma_{0}$ is presented in Fig.2. The capture (for $\gamma_{0}=0.49$ ) and pass-through (for $\gamma_{0}=0.50$ ) phenomena can be observed. 
In the first series of experiments with the algorithm (17), (12) the value of the controlled torque was chosen as $\gamma_{0}=0.2$, which is significantly less than the capture threshold $\gamma_{0}=0.5$. The values of time constant $T$ were tested in the interval from $T=0.1 \mathrm{~s}$ to $T=2 \mathrm{~s}$. The value of $T$ corresponding to the fastest passage through resonance frequency were close to the period of slow motions: $T * \approx 0.7 \mathrm{~s}$. In the second series of experiments we chose $T=0.7 \mathrm{~s}$ and decreased $\gamma$. The results are shown in Fig.3,4 (for $\gamma_{0}=0.26$ and $H^{*}=11$ ).

The simulation results confirm that the algorithm (17), (12) achieves significant reduction of the torque required for passage through resonance

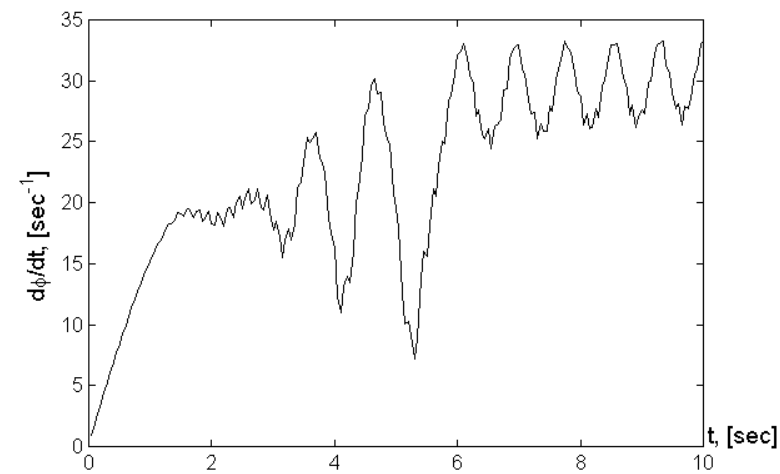

Fig. 3. Controlled passage through resonance, $\gamma_{0}=0.26$.

\section{EXAMPLE 2. VIBRATIONAL UNIT WITH PLANE MOTION OF SUPPORT}

\section{A. Problem statement}

Consider the following system of differential equations describing the plane motion of one-rotor vibrational unit (see Fig. 5) (Blekhman, 2000):

$$
\begin{aligned}
& J \ddot{\varphi}+k_{\varphi} \dot{\varphi}+m \varepsilon(\ddot{x} \cos \varphi+\ddot{y} \sin \varphi+g \sin \varphi)=u(t) \\
& (M+m) \ddot{x}+k_{x} \dot{x}+c_{x} x+m \varepsilon\left(\ddot{\varphi} \cos \varphi-\dot{\varphi}^{2} \sin \varphi\right)=0 \\
& (M+m) \ddot{y}+k_{y} \dot{y}+c y+m \varepsilon\left(\ddot{\varphi} \sin \varphi+\dot{\varphi}^{2} \cos \varphi\right)=M g,
\end{aligned}
$$

where $\varphi$ - rotor angle, $x, y$ - coordinates of the

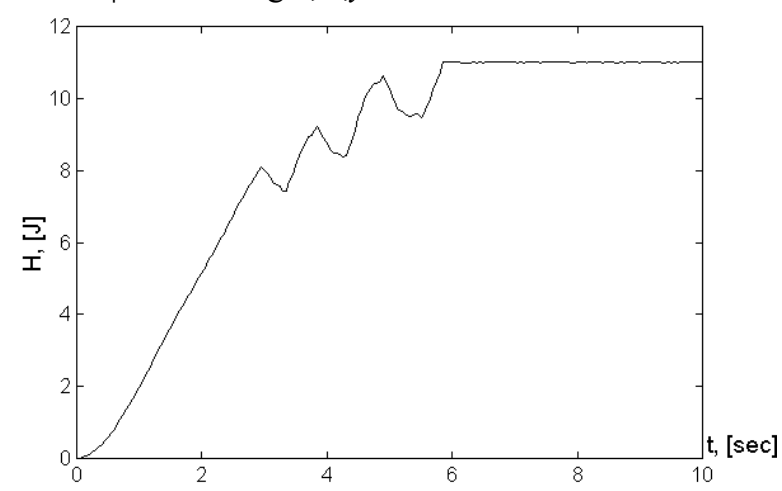

Fig. 4. Energy plot for proposed control: $\gamma_{0}=0.26, H^{*}=11$. platform, $u(t)$ - control action (rotating torque of a motor), $J$ - moment of inertia of an unbalanced rotor (disk), $m$ - mass of a rotor, $M$ - mass of a platform, $\varepsilon$ - eccentricity of the rotor center of mass, $c, c_{x}$ - shaft torsional stiffness, $k_{\varphi}, k_{x}, k_{y}$ damping factors.

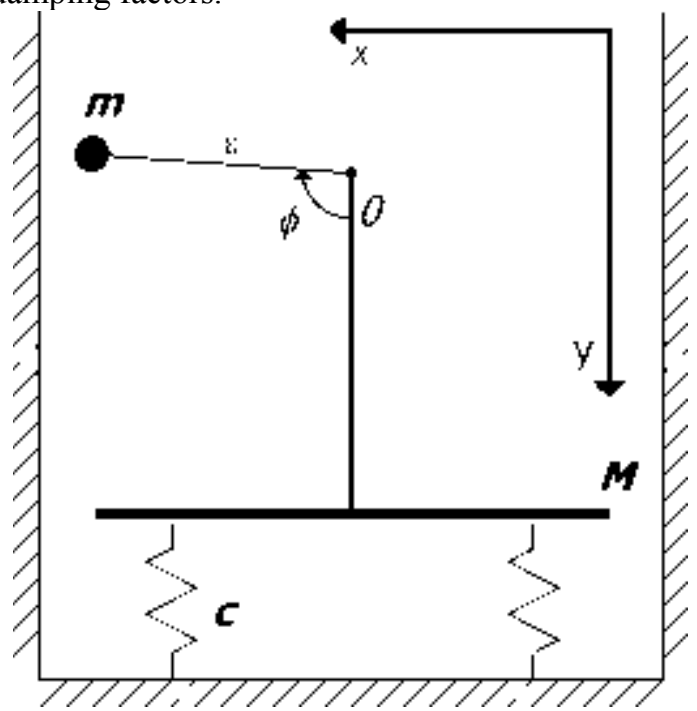

Fig. 5. One-rotor vibrational unit.

It is well-known (Kononenko, 1964), that the "capture" of angular velocity of a rotor (Sommerfeld phenomenon) sometimes takes place in the near-resonance zone. The capture phenomenon happens when the level of constant control action $u(t) \equiv M_{0}$ is small. If the level of constant control action $u(t) \equiv M_{0}$ is higher, the system passes the resonance zone (see Fig. 6) for the nominal values of system parameters: $J=0.014$ $\left[\mathrm{kg} \cdot \mathrm{m}^{2}\right], M=10.5[\mathrm{~kg}], m=1.5[\mathrm{~kg}], \varepsilon=0.04[\mathrm{~m}]$, $k_{\varphi}=0.01 \quad[\mathrm{~J} \cdot \mathrm{sec}], c=5300 \quad[\mathrm{~N} / \mathrm{m}], c_{x}=530$

$[\mathrm{N} / \mathrm{m}], k_{x}=k_{y}=5[\mathrm{~kg} / \mathrm{sec}]$.

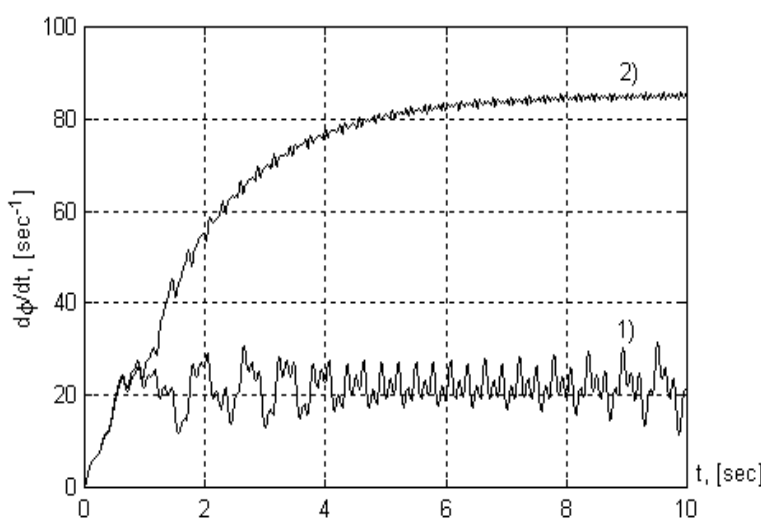

Fig. 6. Angular velocity for conventional control $u(t) \equiv M_{0}$. 1) $M_{0}=0.80$ - capture, 2) $M_{0}=0.81$ - passage.

The problem is to design the control algorithm $u=\boldsymbol{U}(x, \dot{x}, y, \dot{y}, \varphi, \dot{\varphi})$, providing the spin-up of unbalanced rotor until the speed exceeds critical resonant value. The level of control signal is restricted and does not allow the passage through 
resonance when the control signal is constant. The existing optimal control algorithms (Malinin and Pervozvansky, 1993) are hard for implementation and are not sufficiently robust.

\section{B. Design of control algorithms}

To describe the proposed control algorithm first describe the way to define the time of passing through resonance zone. It is proposed to measure the depth of the resonance by the average energy of the rotor center of mass motion, which is proportional to $\left(\dot{x}^{2}+\dot{y}^{2}\right)$. A related measure for oscillatory motion is the average sum of coordinate squares $x^{2}+y^{2}$ which is decreasing when system is passing through the resonance zone. This fact is confirmed by simulation: the sum $x^{2}+y^{2}$ increases when the level of constant control action is small and does not allow system to pass through the resonance zone. In case of higher control torque, the average sum of coordinate squares $x^{2}+y^{2}$ increase in the pre-resonance zone and decrease in the post-resonance zone.

In order to smooth the variable $x^{2}+y^{2}$ we introduce the additional low pass filter:

$$
T_{\theta} \dot{\theta}(x, y, t)=-\theta+x^{2}+y^{2}, \quad \theta(0)=\dot{\theta}(0)=0,
$$

where $T_{\theta}>0, T_{\theta}=$ const - algorithm parameter. The filtered variable $\theta(x, y, t)$ increases when the system is in the pre-resonance or resonance zone. In the post-resonance zone the value of $\theta(x, y, t)$ decreases significantly in comparison with the maximum value. Thus measuring the variable $\theta(x, y, t)$ of the filter (19) allows estimate the time of the passage through resonance. This fact is confirmed by computer simulation, see Fig. 7 .

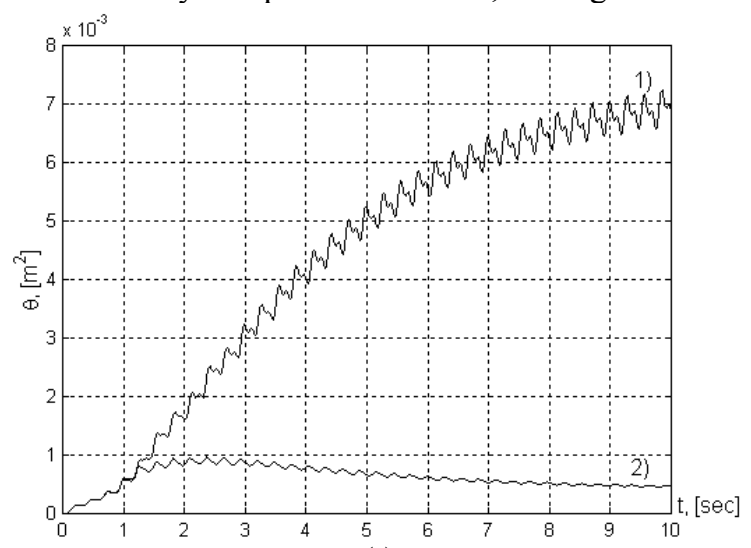

Fig. 7. Plot of $\theta(t)$ for $u(t) \equiv M_{0}$. 1) $M_{0}=0.80$ capture, 2) $M_{0}=0.81$ - passage.

To synthesize the control algorithm we use the speed-gradient method. At this stage we suppose that the control plant is conservative, i.e. the friction equals to zero. The control goal is to find controlling function $u(t)$ providing the goal equality $H(x, \dot{x}, y, \dot{y}, \varphi, \dot{\varphi})=H^{*}$, where $H(\cdot)$ is a current energy, $H^{*}$ is the given energy level corresponding to the desired average rotation speed. Choose the goal functional $Q(z)=1 / 2\left(H(z)-H^{*}\right)^{2}, \quad$ where $z=[x, \dot{x}, y, \dot{y}, \varphi, \dot{\varphi}]^{\mathrm{T}}$. The "relay" form of speedgradient algorithm designed according to the proposed approach is as follows:

$$
\left\{\begin{array}{l}
u=\left\{\begin{array}{l}
M_{0}, \quad \text { if }\left(H-H^{*}\right)(\dot{\varphi}-\psi)>0, \\
0, \quad \text { else, }
\end{array}\right. \\
T_{\psi} \dot{\psi}=-\psi+\dot{\varphi} .
\end{array}\right.
$$

where $\psi(t)$ - filtered variable, $T_{\psi}>0, T_{\psi}=$ const.

However the efficiency of this algorithm is rather low because of high amplitude of rotor oscillations. So the value $H^{*}$ may be achieved in the resonance zone. Also this algorithm requires choosing the value $H^{*}$ for every set of plant parameters, and this task has no evident solution.

Thus we propose to exclude the factor $H-H^{*}$ having the negative sign in the post-resonance zone, from the algorithm. We also propose to switch off the control in the post-resonance zone, keeping only constant control torque. The algorithm is modified as follows.

The variable $\gamma_{1}(t)$ :

$$
\gamma_{1}(t)=\max _{[0, t]} \operatorname{sgn}\left[K \sup _{[0, t]} \theta(t)-\theta(t)\right],
$$

is introduced, where $K>0$ is the algorithm parameter. The properties of the variable $\theta(t)$ allows to say that $\gamma_{1}(t)=0$ means that the system is in the pre-resonance or resonance zone (there was no significant decrease of $\theta(t))$. Also $\gamma_{1}(t)=1$ means that the system is in the postresonance zone. Thus, $\gamma_{1}(t)$ characterizes the current behavior of the system if $K$ is properly chosen. The value of $K$ should be sufficiently small to guarantee that the system is already in the postresonance zone. At the same time the unjustified decrease of $K$ may reduce the efficiency and transient time of the proposed algorithm.

Finally, the algorithm takes the form:

$$
\left\{\begin{array}{l}
u(t)=\left\{\begin{array}{l}
M_{0}, \quad \text { if } \quad \gamma_{1}(t)=1, \\
M_{0}, \quad \text { if } \quad \gamma_{1}(t)=0 \quad \& \quad(\dot{\varphi}-\psi)<0 \\
0, \quad \text { else, }
\end{array}\right. \\
T_{\psi} \dot{\psi}=-\psi+\dot{\varphi}, \\
\gamma_{1}(t)=\max _{[0, t]} \operatorname{sgn}\left[\begin{array}{c}
K \sup \theta(t)-\theta(t) \\
{[0, t]}
\end{array}\right] \\
T_{\theta} \dot{\theta}(t)=-\theta(t)+x^{2}+y^{2}, \quad \theta(0)=\dot{\theta}(0)=0
\end{array}\right.
$$


The value of $T_{\psi}$ (time constant of the angular velocity filter) should be more then the period of the resonant oscillations. At the same time, if the value of $T_{\psi}$ is too high, the algorithm works too slowly.

\section{Simulation Results}

The designed control algorithm was numerically investigated to analyze the efficiency of the proposed algorithm. Numerical integration was made in MATLAB environment by means of Runge-Kutta method of second order. The value of the fixed step equal to 0.00025 [sec] was chosen so as the relative simulation error does not exceed $5 \%$. The nominal values of system parameters were chosen as in (Blekhman, et. al., 1999): $J=0.014$ $\left[\mathrm{kg} \cdot \mathrm{m}^{2}\right], M=10.5[\mathrm{~kg}], m=1.5[\mathrm{~kg}], \varepsilon=0.04[\mathrm{~m}]$, $k_{\varphi}=0.01 \quad[\mathrm{~J} \cdot \mathrm{sec}], c=5300 \quad[\mathrm{~N} / \mathrm{m}], c_{x}=530$ $[\mathrm{N} / \mathrm{m}], k_{x}=k_{y}=5[\mathrm{~kg} / \mathrm{sec}]$.

It is seen that the value of the rotating torque of a motor, allowing system to pass the resonance zone for $u(t) \equiv M_{0}$, is reduced in almost two times by means of the proposed algorithm.

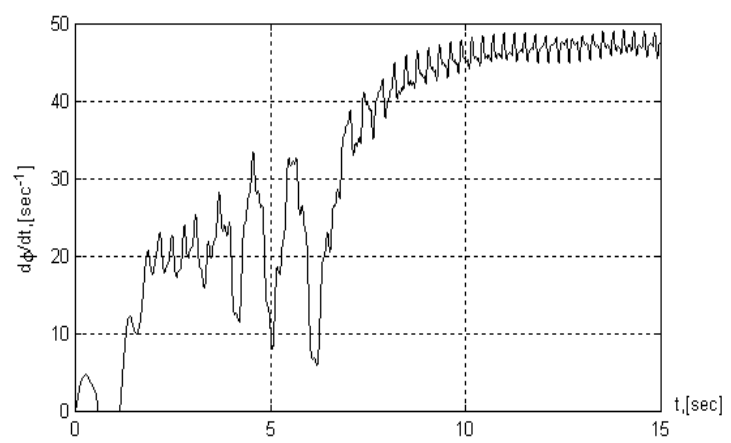

Fig. 8 Angular velocity of controlled system, $M_{0}=0.46$.

\section{CONCLUSIONS}

New algorithms of passing through resonance zone for mechanical systems are proposed and analyzed by computer simulation. The algorithms are based on speed-gradient method and allow to significantly reduce the required level of the controlling torque. The algorithms have small number of design parameters and, compared with the known algorithms are more simple for design. It is planned to test the proposed algorithm on the two-rotor vibrational set-up (Blekhman, et. al., 1999).

\section{ACKNOWLEDGEMENTS}

The work was supported by RFBR grant 05-0100869.

\section{REFERENCES}

Blekhman, I.I., O.L.Nagibina, O.P. Tomchina and K.S. Yakimova (1997). Control of oscillations in electromechanical systems. Proc.Int.Conf. on Informatics and Control, St.Petersburg, pp.972-979.
Blekhman, I.I., Yu.A.Bortsov, A.A.Burmistrov, A.L.Fradkov, S.V.Gavrilov, O.A.Kononov, B.P.Lavrov, V.M.Shestakov, P.V.Sokolov and O.P.Tomchina. (1999). Computer-controlled vibrational set-up for education and research. Proc. 14 ${ }^{\text {th }}$ IFAC World Congress, vol...M, pp.193-197.

Blekhman, I.I. (2000). Vibrational mechanics. World Scientific.

Bupp, R.T. Bernstein, D.S., Coppola, V.T. (1998) A benchmark problem for nonlinear control design," Int. J. Robust Nonlinear Contr., vol. 8 pp. 307-310.

Evan-Iwanowski, R.M. (1976). Resonance oscillations in mechanical systems. Elsevier, Amsterdam.

Fradkov, A.L. (1996). Swinging control of nonlinear oscillations. Int. J. of Control, 64 (6), pp. 1189-1202.

Fradkov, A.L., I.A. Makarov, A.S. Shiriaev and O.P. Tomchina (1997). Control of oscillations in Hamiltonian systems. Proc. 4th European Contr.Conf. Brussels, TH-M-G-6, 1997.

Jankovic, M., D. Fontaine and P.V. Kokotovic (1996). TORA example: Cascade and passivity based designs. IEEE Trans. Control Systems Technology, v. 4, pp. 292-297.

Kel'zon, A.S. and L.M. Malinin (1992). Control of rotor oscillations. Politekhnika, Saint Petersburg (in Russian).

Kinsey, R.J., D.L.Mingori and R.H.Rand. (1992). Nonlinear controller to reduce resonance effects during despin of a dual-spin spacecraft through precession phase lock. Proc. $31^{\text {th }}$ IEEE Conf.Dec.Contr, 1992, pp. 3025-3030.

Kononenko, V.O. (1964). Oscillatory systems with bounded excitation. Nauka, Moscow (in Russian).

Malinin, L.N. and A.A.Pervozvansky (1993). Optimization of passage an unbalanced rotor through critical speed. Mashinovedenie, 4, pp.36-41 (in Russian).

Pechenev, A.V. (1986). About motion of oscillating system with bounded excitation near resonance. Physics-Doklady, v.32 (Doklady Academii Nauk SSSR, vol.290, No.1)

Rand, R.H., R.J. Kinsey and D.L. Mingori (1992). Dynamics of spinup through resonance. Intern. Journal of Nonlinear Mechanics, vol. 27, no.3, pp.489-502.

Tomchina, O.P. (1997). Passing through resonances in vibratory actuators by speedgradient control and averaging. Proc.Int.Conf. Control of Oscillations and Chaos, IEEE, St.Petersburg, v.1, pp.138-141.

Tomchina, O.P. and K.V. Nechaev (1999). Controlling passage through resonances in vibratory actuators. Proc. $5^{\text {th }}$ European Contr. Conf.,Karlsruhe, Sept.1999.

Wan, C.-J., D.S. Bernstein and V.T. Coppola (1994). Global stabilization of the oscillating eccentric rotor. Proc. 34th IEEE Conf.Dec.Contr., pp.4024-4029. 